\title{
PRESENTACIÓN PÚBLICA DE LA VERSIÓN ACTUAL Y RESEÑA HISTÓRICA DE LA REVISTA PERUANA DE MEDICINA EXPERIMENTAL Y SALUD PÚBLICA
}

\section{PUBLIC PRESENTATION OF THE UPDATED VERSION AND HISTORICAL REVIEW OF REVISTA PERUANA DE MEDICINA EXPERIMENTAL Y SALUD PÚBLICA}

\author{
Zuño Burstein Alva*
}

\begin{abstract}
El 26 de enero de 2011, en el auditorio del Instituto Nacional de Salud, se realizó la ceremonia de presentación pública de la Revista Peruana de Medicina Experimental y Salud Pública último número del año 2010, publicación que es el órgano oficial de difusión científica del Instituto Nacional de Salud, brazo científico del Ministerio de Salud del Perú.
\end{abstract}

La presentación de la Revista se desarrolló en el marco de una ceremonia conjunta del Instituto Nacional de Salud y la Academia Nacional de Ciencias, programada en homenaje a la destacada personalidad médica del Dr. Carlos Gutiérrez Noriega que, entre otros reconocidos méritos, fue fundador de nuestra Revista el año 1942, junto al Dr. Telémaco Battistini ${ }^{(1-2)}$.

Concurrieron a la ceremonia diversas personalidades en representación de la Academia Nacional de Ciencias, la Academia Nacional de Medicina, la Academia Peruana de Salud, la Academia Peruana de Cirugía, la Universidad Nacional Mayor de San Marcos y la Universidad Peruana Cayetano Heredia; así también, editores de revistas médico-científicas, miembros del Consejo Consultivo de la Revista, personal que colaboró en la edición de la Revista y miembros de la institución anfitriona.

La ceremonia se inició con las palabras introductorias y de saludo del Subjefe del Instituto Nacional de Salud, Dr. Luis Santa María, quien destacó la importancia de la investigación médico - científica aplicada a la Salud Pública y su adecuada difusión informativa. Luego, el Dr. Pedro Álvarez Falconí, Médico Farmacólogo, miembro del Comité Editor de la Revista y relacionado profesionalmente con el homenajeado, hizo una emotiva semblanza donde resaltó la importante vinculación de este notable investigador con el Instituto Nacional de Higiene y Salud Pública (primer nombre de esta Institución) quien falleciera trágicamente en Italia, el año 1950.

En el acto central de la ceremonia y de acuerdo con lo programado, el Doctor Roger Guerra- García, Presidente de la Academia Nacional de Ciencias, hizo la presentación del libro "Cultura y personalidad; escritos conexos", de Carlos Gutiérrez Noriega, compilado por el Dr. Guerra-García y por el Dr. Javier Mariátegui Chiappe ( $\dagger$ ), este libro fue coeditado por la Academia Nacional de Ciencias y el Fondo Editorial de la Universidad Nacional Mayor de San Marcos, del cual es Director, el Dr. Gustavo Delgado Matallana, y quien posteriormente intervino con palabras alusivas al homenaje.

Acto seguido se realizó la presentación de la Revista, con la participación, en primer lugar, del director Dr. Zuño Burstein, quien se encargó de realizar una reseña y un análisis de la historia de nuestra publicación. A continuación el Dr. Percy Mayta -Tristán, Editor Científico de la Revista, hizo una bien documentada exposición técnica del proceso de edición de la Revista, señaló los cambios y mejoras en los recursos humanos y materiales que, mediante una adecuada dinámica organizativa administrativa y de dirección, así como la adopción de una política editorial comprometida, conservando una gran exigencia en los artículos publicados, han permitido que la Revista, además de su gran impacto, haya sido calificada e incorporada en las más exigentes bases de datos, lo cual nos ubica a la altura de las

* Director de la Revista Peruana de Medicina Experimental y Salud Pública.

Correo electrónico: zburstein_2000@yahoo.com 
más prestigiosas publicaciones médico - científicas en idioma español, ello representa un orgullo para el Instituto puesto que es la única entidad estatal que tiene este privilegio en el ámbito latinoamericano, a su vez, este estado privilegiado representa una valiosa tarjeta de presentación y de posicionamiento del Perú en el mundo científico de nuestra región y a nivel global.

\section{HISTORIA DE LA REVISTA PERUANA DE MEDICINA EXPERIMENTAL Y SALUD PÚBLICA}

Para tener una idea adecuada de lo que hoy es la Revista, es necesario referir los antecedentes del nombre y la evolución histórica de la publicación oficial en la institución que le dio origen. La Revista inició su publicación en octubre de 1942 con el nombre de Revista de Medicina Experimental, Volumen 1 Números 1 y 2, este primer número fue editado por los doctores Carlos Gutiérrez Noriega y Telémaco Battistini, y tuvo una periodicidad trimestral hasta el año 1960, como órgano oficial de difusión científica del Instituto Nacional de Higiene y Salud Pública.

En 1946 se constituye su Comité Editor con las siguientes personas: Director: Dr. Telémaco Battistini; Secretario de Redacción: Dr. Oscar Miro Quesada; Comité de Redacción: doctores Celso Arellano, Víctor Ayulo, Germán Battistini, Aristides Herrer, Julio Pons M., Oscar Rondón, Pedro Weiss y Vicente Zapata. Se incorporan posteriormente los doctores Luis Gonzales Mugaburu, José Madalengoitia, René Solís, Julio Morales, Felipe Llanos, Jorge Díaz y otros.

Luego de esa época de oro, la Revista de Medicina Experimental siguió un prolongado silencio editorial que duro 37 años, en cuyo interin se editó, en reemplazo de la Revista, otro órgano oficial de difusión de la institución, con carácter de comunicación técnico - científica, con el nombre inicial de Boletín de los Institutos Nacionales de Salud ${ }^{(3)}$, que continuó su publicación impresa hasta el año 2009.

\section{Boletín de los Institutos Nacionales de Salud}

Inicia su publicación en 1980 con un primer número correspondiente a enero-marzo (3), bajo la dirección del Dr. Germán Battistini y como Editor Responsable el Dr. Luis Gonzales Mugaburu, apoyados por el Comité de Biblioteca y Publicaciones integrado por los doctores Juan Arbaiza, Zuño Burstein, Arístides Herrer, Bertha Llanos, Enrique Morales, Rolando Urbina, Enrique Vidarte, Soledad Urquizo, Carmen Piedra y la Bibliotecaria Sra. María de Betalleluz.
En años ulteriores, otros profesionales de la Institución se sumaron al esfuerzo de mantener vigente la edición impresa del Boletín en el que se publicaron importantes trabajos originales de investigación, realizados por los investigadores del INS. Destacan en esa responsabilidad editorial los doctores Alberto Cuba Caparó, Pedro Álvarez Falconi, César Cabezas y últimamente Javier Vargas, que mantuvo durante varios años la presencia de este órgano oficial de difusión institucional que recientemente vino a ser reemplazado con la versión electrónica informativa semanal del INS.

El año 1997 se reinicia la publicación de la Revista de Medicina Experimental - Segunda Época ${ }^{(4)}$, con el volumen XIV, correspondiente al año 1997 y el volumen $\mathrm{XV}$ al año 1998 , con los que se conmemoraba el $61 .^{\circ} \mathrm{y}$ $62 .^{\circ}$ Aniversario de la Institución (1936-1997) y (19361998), respectivamente; se encontraba en la dirección del Instituto el Dr. Carlos Carrillo y como responsables en la edición el Dr. César Cabezas y el Dr. César Náquira, a los que se agregaron, desde 1999 y en años sucesivos, los doctores Zuño Burstein, Pedro Álvarez, Aída Palacios (Como Presidenta del Comité Editor), Alfredo Guillén entre, otros; en la responsabilidad administrativa estuvo como Editor el Dr. Leonid Lecca. El año 2001 la Revista cambia de nombre por el actual.

\section{Fundamentación para el cambio de nombre}

En la nota Editorial del Vol.19 (1) del año 2002 se expone lo siguiente: El esfuerzo editorial iniciado en 1942 con el nombre de Revista de Medicina Experimental se refleja en 30 números publicados hasta el año 2001; originalmente se aceptaban trabajos en microbiología, fisiología, patología, farmacología o higiene $y$, desde 1997, se amplía el contenido de los artículos publicados, abarcando áreas temáticas del campo de la salud pública. Esto fundamenta el cambio de nombre de esta publicación a Revista Peruana de Medicina Experimental y Salud Pública y formaliza los cambios en los contenidos de los artículos publicados, incluyendo todas las áreas relacionadas con la salud pública, como nutrición, salud ocupacional, protección del medio ambiente, salud intercultural, administración pública y sanitaria, antropología, política en salud, accidentes y desastres, salud mental, bioterrorismo, biología molecular, aseguramiento de la calidad, control de calidad de alimentos y medicamentos y otros temas de interés.

Para completar la información relacionada con la evolución histórica de la Revista, es necesario conocer la historia de los cambios de nombre y estructura del Instituto Nacional de Salud INS/MINSA a través del tiempo, materia que será publicada en un próximo número de la Revista. 


\section{Análisis crítico y cronología de la Revista}

Haciendo un análisis critico de la evolución cronológica de la Revista, correlacionándola desde su fundación hasta la fecha, con los periodos correspondientes a las sucesivas jefaturas en el Instituto, a los cargos ministeriales y a las presidencias del Gobierno Nacional, constatamos, entre otras ocurrencias, que el prolongado silencio editorial de nuestras publicaciones se dio durante los gobiernos militares; mientras que la exitosa situación lograda durante estos últimos siete años se debe al apoyo comprometido de quienes ocuparon la jefatura del Instituto como son los doctores Fernando Llanos, César Náquira, Patricia García, Aníbal Velásquez y, actualmente del Dr. César Cabezas, vinculado históricamente con la Revista; del mismo modo ocuparon el cargo de Ministros de Salud durante esa época los doctores Álvaro Vidal, Pilar Mazzetti, Carlos Vallejos y actualmente el Dr. Oscar Ugarte; en los gobiernos presidenciales de Alejandro Toledo y Alan García.

\section{REVISTA PERUANA DE MEDICINA EXPERIMENTAL Y SALUD PÚBLICA ESTADO ACTUAL}

La Revista Peruana de Medicina Experimental y Salud Pública, órgano oficial de difusión científica del Instituto Nacional de Salud del Ministerio de Salud del Perú, es una tribuna de expresión abierta para la publicación de contribuciones sobre el avance de la medicina en el área de su competencia, de las investigaciones que realiza nuestra institución y de las comunicaciones que puedan aportar los miembros del equipo de salud del país, así como las que nos envían autores e instituciones extranjeras vinculadas con los problemas de la salud pública del Perú.

Nuestra Revista recibe, a través de notas editoriales, artículos originales, temas de revisión, comunicaciones cortas, reportes de casos, galerías fotográficas, cartas al editor y una sección especial, los aportes y controversias relacionados con nuestra responsabilidad informativa, previa revisión por pares.

Se ha establecido como política editorial desarrollar en cada número de la revista o, si es del caso, en varios números consecutivos, un tema prioritario relacionado con la salud pública del Perú, tal como se ha llevado a cabo hasta el momento con los siguientes temas: Rabia, Desnutrición Crónica, Infecciones de Transmisión Sexual, Hepatitis B, Educación Médica, Medicina de Emergencia y Desastres, Salud Ambiental, Política de Medicamentos, Tuberculosis, Accidentes de Tránsito, Interculturalidad en Salud, Política en Investigación en Salud y Zoonosis Parasitarias .

Así mismo, se ha designado a un Editor Invitado, quién es un reconocido investigador en el tema por tratar, para coordinar la captación de artículos y responsabilizarse de esta misión, que en coordinación con nosotros, se ocupa del tema correspondiente. Estos artículos se incorporarán a la revista en sus diferentes secciones 0 , si es del caso, bajo una forma de Simposio.

Agradecemos la valiosa colaboración de los siguientes profesionales que actuaron como Editores Invitados durante estos últimos números, los doctores Raúl Morales Soto, Hugo Rengifo, Pedro Álvarez, Aníbal Velásquez, Eduardo Ticona, Oswaldo Salaverry, Jaime Miranda, Martin Yagui y César Náquira.

Las contribuciones comprometidas para el Simposio por el Editor Invitado no siguen el mismo rigor que los artículos que forman parte de las otras secciones de la Revista. Se deja, entonces, en libertad para una participación que aporte valiosa información al tema que es motivo del Simposio. En estos casos, el Comité Editor asume la responsabilidad de coordinar con los autores y con el Editor Invitado para hacer la adecuación pertinente y lograr el nivel que corresponde a nuestra publicación.

Desde julio de 2003, el Dr. Zuño Burstein asume la Dirección de la Revista y se incorporan al Comité Editor del INS representantes de los diferentes Centros además de miembros externos a la Institución; asume la función de Editor Asociado el biólogo Rufino Cabrera, acompañan al Editor la bibliotecóloga Mg. Graciela Rengifo y el Lic. Daniel Cárdenas-Rojas (que venia ejerciendo como Asistente Editorial desde el año 2002). Desde el año 2004 el Dr. Percy Mayta Tristán asume el cargo de Editor Científico de la Revista (cargo que desarrolla con gran competencia profesional), años después y lo acompaña el Dr. Edward Mezones-Holguín como Editor Adjunto, se incorporó, también al Comité Editor a los doctores Javier Vargas y Oswaldo Salaverry, entre otros.

En estos últimos seis años se ha logrado, gracias al apoyo de los niveles jerárquicos correspondientes y la identificación de los colaboradores comprometidos con la Revista, una adecuada formalización e individualización de la estructura organizativa de la Revista, la cual está conformada por *:

1. Un nivel de Dirección con un Director, un Editor General, un Editor Científico y uno a dos Editores Adjuntos a cargo de los Drs. Zuño Burstein , César Cabezas, Percy Mayta-Tristán y Edward MezonesHolguín, respectivamente.

2. Un Comité Editor conformado por distinguidos profesionales del Instituto y miembros externos a la Institución, con reconocido nivel académico y experiencia editorial cuya nómina figura en la Revista. 
3. Un Consejo Consultivo integrado por personalidades científicas del país y del extranjero, invitados a conformar este importante estamento y cuya nómina figura en la Revista.

4. Un nivel de apoyo técnico administrativo, a cargo de:

4.1 Un Coordinador Administrativo, cargo que estuvo desempeñado sucesivamente por el Lic. José Villegas y Mónica Suárez, Jefes de la oficina General de Información y Sistemas del INS.

4.2 Un encargado de la distribución de la Revista a cargo de la bibliotecóloga Mg. Graciela Rengifo, Directora Ejecutiva de la Oficina de Información y Documentación Científica del INS.

4.3 Una Asistente Editorial, cargo desempeñado sucesivamente por las Lics. Melisa Daga del Castillo, Carolina Tarqui y Bertha Huarez; y un Corrector de Estilo a cargo del Lic. Daniel Cárdenas-Rojas.

El competente apoyo de este nivel técnico administrativo a la Dirección y a los Editores de la Revista ha permitido alcanzar el alto grado de excelencia obtenido en estos últimos años. Hay que mencionar la valiosa colaboración de la Secretaria, Sra. Olga Otárola, y del recientemente incorporado Técnico Administrativo, Sr. Aníbal Espinoza, destacados para apoyar al Comité de la Revista.

Para este año 2011 se ha programado cuatro números del volumen 28 , los cuales serán publicados en su debida oportunidad de acuerdo con una rigurosa calendarización, los siguientes temas de interés y prioritarios en salud pública son: Enfermedades diarreicas, para este primer número; para los siguientes son: Recursos humanos en salud, Evaluaciones económicas en salud y Ética y medicina; para los cuales ya se encuentran comprometidos, para cada uno de estos temas respectivamente, a los siguientes Editores Invitados: Dr. Alfredo Guillén, Médico Microbiólogo, Profesor de la Facultad de Tecnología de la Universidad Nacional Federico Villarreal; Dr. Luis Huicho, Médico
Pediatra, profesor principal de la Universidad Nacional Mayor de San Marcos; Dr. Edward Mesones, Médico Epidemiólogo, Investigador de la Unidad de Generación y Análisis de Evidencias en Salud Pública INS/MINSA, Profesor de Bioestadística de la Universidad Peruana de Ciencias Aplicadas y el Dr. Alberto Perales, Médico Psiquiatra, Profesor Principal de la Universidad Nacional Mayor de San Marcos, Vicepresidente de la Academia Nacional de Medicina. Todos ellos de reconocida competencia profesional y académica, y expertos en el tema a su cargo, todo ello nos permitirá, con la contribución de destacados autores y colaboradores con trabajos ya en nuestra carpeta de publicaciones, mantener nuestra calificación en el alto nivel logrado como una de las revistas médico - científicas más prestigiadas en el idioma español y la única de estas características de origen estatal.

Finalmente, en el contexto de nuestra responsabilidad en el conocimiento y toma de decisiones en salud pública, hemos introducido el procedimiento de revisión rápida en casos de situaciones de emergencia sanitaria. Ello ha permitido ofrecer en el presente número, dos artículos de aporte relacionados con el reciente brote epidémico de dengue y la caracterización del nuevo serotipo circulante, además de un interesante reporte concerniente a la presentación de un caso de dengue neonatal.

\section{REFERENCIAS BIBLIOGRÁFICAS}

1. Burstein, Z. Telémaco Battistini Sánchez, (18951960). Rev Peru Med Exp Salud Publica. 2010; 27(1):142-45.

2. Enciclopedia Biográfica e Histórica del Perú Siglo XIX-XX. Lima: Editorial Milla Batres; 1994.

3. Instituto Nacional de Salud. Bol Inst Nac Salud. Lima; INS; 1980;1(1):1-32.

4. Instituto Nacional de Salud. Revista de Medicina Experimental. Segunda época. Lima: INS; 1997:Vol XIV (1) y (2).

* El Comité Editor de la Revista Peruana de Medicina Experimental y Salud Pública incluyendo los editores científico y adjunto, así como el comité consultivo han sido recientemente reestructurados. La nueva conformación puede verse en la página inicial de este número. 